\title{
REPRODUCCIÓN INDUCIDA DE LA DONCELLA \\ Pseudoplatystoma fasciatum y DESARROLLO \\ EMBRIONARIO - LARVAL
}

\section{Palmira P. Padilla Pérez*, Fernando Alcántara Bocanegra*, Rosa Ismiño Orbe*}

\section{RESUMEN}

Los productos sexuales de doncella Pseudoplatystoma fasciatum fueron obtenidos mediante la inducción hormonal usando pituitaria de carpa y ovudal (D-alanina 6). La dosis de ovudal fue de $10 \mathrm{mg} / \mathrm{kg}$ para las hembras y de $1 \mathrm{mg} / \mathrm{kg}$ para los machos, mientras que la de pituitaria de carpa fue de $5 \mathrm{mg} / \mathrm{kg}$ para las hembras y $1 \mathrm{mg} / \mathrm{kg}$ para los machos.

Los huevos son esféricos, semiflotantes y no adhesivos. Están protegidos externamente por una capa gelatinosa e, internamente, por un gran espacio perivitelínico. El diámetro de los huevos hidratados es de $8,5 \mu$.

La eclosión se inició a las $14 \pm 2$ horas de incubación a $27^{\circ} \mathrm{C}$ de temperatura. Al nacer, las larvas tienen una longitud total de $3,02 \pm 0,12 \mathrm{~mm}$ y presentan un saco vitelino. Después de 21 horas, se observaron los pigmentos oculares y comenzaron a desarrollarse las barbillas maxilares. A los 3 días, el saco vitelino se reabsorbe y se completa la conexión del tubo digestivo con la boca. A partir del cuarto día, las larvas consumen alimento vivo y tienen una longitud total de 5,82 $\pm 0,19 \mathrm{~mm}$.

En el décimo día, se define el primer patrón de coloración. En este periodo, las larvas tienen una longitud total de $10,7 \pm 0,4 \mathrm{~mm}$.

Palabras clave: Reproducción inducida, Pseudoplatystoma fasciatum, desarrollo embrionario, larvas.

* Instituto de Investigaciones de la Amazonía Peruana. Programa de Ecosistemas Acuáticos. Centro Regional de Investigaciones de Loreto. Apartado Postal 1784, Iquitos (Perú). Teléfonos: 094-265515 - 265516. Fax :094-265527. 


\begin{abstract}
Were obtained sexual products of «Doncella» Pseudoplatystoma fasciatum, by means of the induction, using pituitary of «Carpa» and ovudal (D-alanina 6). The dose of ovudal was $10 \mathrm{mg} / \mathrm{kg}$ for females and $1 \mathrm{mg} / \mathrm{kg}$ for males and with pituitary of «Carpa», the dose was $5 \mathrm{mg} / \mathrm{kg}$ for females and $1 \mathrm{mg} / \mathrm{kg}$ for males. The eggs are spheric, semifloating, not adhesive, protected externally by a layer gelatin and internally with a great perivitelinic space. The diameter of the hydrated eggs is of $8,5 \mu$.

The eclosion begun after $14 \pm 2$ hours of incubation $27^{\circ} \mathrm{C}$ of temperature. The larvae upon being born have a total length of $3,02 \pm 0,12 \mathrm{~mm}$ and they present bag vitelino. After 21 hours were observed the ocular pigments and then begin to be developed the maxillars beard. After 3 days the bag vitelino themselves reabsorb and themselves complete the connection of the digestive pipe with the mouth. As of the fourth day the larvae consume alive food and they have a total length of $5,82 \pm 0,19 \mathrm{~mm}$.

In the tenth day the first boss of coloring is defined. In this period have a total length of $10,7 \pm 0,4 \mathrm{~mm}$.
\end{abstract}

Key words: Induced reproduction, Pseudoplatystoma fasciatum, embryonic development, larvae.

\title{
1. INTRODUCCIÓN
}

La ictiofauna amazónica está compuesta en su mayoría de peces considerados en los órdenes characiformes, siluriformes, perciformes y osteoglosiformes. Estos órdenes constituyen importantes grupos de peces que desempeñan un rol particular como componentes de los desembarques en los principales puertos de la cuenca.

La composición de las capturas se debe, entre otras razones, a la abundancia y a la preferencia por ciertas especies. Pero, en cualquier caso, las especies preferidas reciben mayor presión de pesca. Las especies más requeridas por su buena aceptación en el mercado, como Colossoma macropomum, Piaractus brachypomus, Arapaima gigas y los grandes bagres como el Pseudoplatystoma fasciatum, fueron fuertemente cazadas. La recuperación de sus poblaciones no será una tarea fácil por lo difícil que resulta controlar las capturas y la creciente demanda. Ante tal situación, la alternativa es la producción a través de la piscicultura. Para ello, se necesitan varias actividades, como es el cultivo, la alimentación, el manejo de estanques, la reproducción induci- 
da, etc. Este camino podría ayudar a satisfacer la demanda sin presionar los stocks naturales.

Pseudoplatystoma fasciatum tiene carne con excelente sabor. El gran tamaño que alcanza ha despertado, en estos últimos años, un creciente interés por este pez en la piscicultura. Además, está considerado como un pez ornamental y está entre las especies que presenta mayor demanda en los mercados de Colombia, Venezuela y Brasil (Lopes et al., 1996). Su reproducción inducida a través de la aplicación de inyecciones de hormonas viene siendo realizada con relativo éxito por Kossowski y Madrid, 1985; Contreras y Contreras, 1989; Cancino, 1990 y Castagnolli, 1992. El presente trabajo constituye el primero en la Amazonía Peruana en relación con este campo y esta especie.

El objetivo del presente ensayo fue realizar trabajos de reproducción inducida de la doncella, Pseudoplatystoma fasciatum, así como también observaciones de su desarrollo embrionario-larval.

\section{MATERIALES Y MÉTODOS}

El experimento se realizó en el Centro Regional de Investigación (CRI) Loreto del Instituto de Investigaciones de la Amazonía Peruana.

Antes del tratamiento hormonal, se seleccionó a las hembras que presentaron el vientre más prominente y a los machos que emitían esperma por presión del vientre. Para observar el grado de maduración de los óvulos, se extrajo del ovario, introduciendo una sonda plástica por el oviducto, una muestra ellos. Luego de la selección, se trasladaron los peces en hamacas de tela hasta los estanques de tratamiento hormonal. En cada estanque, se colocaron dos especímenes: una hembra y un macho, separados por una malla.

Diez peces fueron sometidos al tratamiento hormonal, cuatro de ellos con extracto de hipófisis de carpa, Cyprinus carpi, y seis, con ovudal (D-alanina 6). La proporción de sexos fue de 1:1. La hormona se aplicó en una sola dosis, calculada de acuerdo con el peso de cada ejemplar (Woynarovich, 1998; comunicación pers.).

Para disolver el ovudal, se usó $2 \mathrm{ml}$ de suero fisiológico. La dosis fue de $10 \mathrm{mg} / \mathrm{kg}$ para las hembras y de $1 \mathrm{mg} / \mathrm{kg}$ para los machos. Cuando se usó pituitaria de carpa, la dosis fue de $5 \mathrm{mg} / \mathrm{kg}$ para las hembras y de $1 \mathrm{mg} / \mathrm{kg}$ para los machos. Para inocular las hormonas, se usó jeringas hipodérmicas de $3 \mathrm{ml}$ con una aguja para insulina. El 
área de inoculación fue el espacio que está entre la aleta abdominal y la papila urogenital.

El momento del desove fue determinado al observarse la liberación de óvulos en el estanque. Seguidamente, se extrajo a la hembra y se le secó con una toalla todo el cuerpo; luego se le presionó el abdomen suavemente. Finalizada la extrusión, se pesaron los óvulos obtenidos, y se determinó el número de óvulos por gramo y el número total de óvulos. Con estos datos, se elaboró la gráfica de la relación entre el peso de cada hembra. Por lo que respecta a los machos, se siguió el mismo procedimiento. La fecundación se realizó en seco (Woynarovich y Horvath,1981; Woynarovich y Woynarovich, 1998). Ver Figura 1, Tabla 1 y Gráfico 1.

La incubación de los huevos se realizó en incubadoras cilindro cónicas de 60 litros de capacidad (Bermúdez et al., 1979; Woynarovich y Horvath, 1981; Alcántara y Guerra, 1986). En cada una de estas, se colocaron $350 \mathrm{ml}$ de huevos ya hidratados. El flujo del agua de incubación fue de 0,3 1/min. Durante la incubación se registraron los datos de temperatura $\left({ }^{\circ} \mathrm{C}\right)$, oxígeno disuelto $(\mathrm{mg} / \mathrm{l})$ y $\mathrm{pH}$. (Tabla 2$)$.

La descripción del desarrollo embrionario de la especie fue realizada hasta el momento de la eclosión. Para facilitar la descripción del desarrollo larval, 100 larvas recién nacidas se pusieron en un acuario de vidrio de 60 litros de capacidad. Esta descripción se basó en la observación de tres ejemplares diarios (de un total de 30 ejemplares). A partir de la absorción del saco vitelino, los ejemplares fueron alimentados con zooplancton (daphnias, ciclops, etc.) y cápsulas de huevos de ave.

Las observaciones del desarrollo embrionario y larval fueron realizadas usando un microscopio binocular LEITZ-WETZLAR con aumento de 100x. Ver Figura 2 y Figura 3.

El número de larvas obtenidas se estimó de acuerdo con la fórmula de Rothbard (1981):

$$
\begin{array}{cl}
\frac{\mathrm{K}}{\mathrm{V} \sum \mathrm{ni}=\mathrm{N}} \quad \text { donde: } & \mathrm{K}=\text { Número de muestras }(\mathrm{K}>1) \\
\mathrm{K} & \mathrm{ni}=\text { Número de larvas en la muestra } \\
\mathrm{V} & =\text { Volumen del recipiente de larvas } \\
& \mathrm{N}=\text { Número total de larvas }
\end{array}
$$




\section{RESULTADOS}

Los especímenes tratados respondieron favorablemente al tratamiento de inducción hormonal. Se produjo el desove a las $20 \pm 4 \mathrm{~h}$ a una temperatura de $25,5 \pm 0,4^{\circ} \mathrm{C}$. Se encontró que las doncellas hembras de $3,570 \pm 1,580 \mathrm{~kg}$ tienen $444,000 \pm 40,830$ óvulos, con una media de 1,204 \pm 8 óvulos por gramo de óvulos. El peso de las hembras y el número de huevos muestran una correlación lineal positiva del tipo $\mathrm{Y}=\mathrm{a}+\mathrm{bx}$, con un coeficiente menor que su valor crítico $(\mathrm{P}=0,05) ; \mathrm{r}=0,701144 ; \mathrm{Y}=14604+120 \mathrm{X}$. Ver Figura 1, Tabla 1 y Gráfico 1.

Figura 1. Pasos de la liberación y fertilización de los óvulos. I. Liberación de los óvulos; II. Liberación del esperma; III. Fertilización de los óvulos.

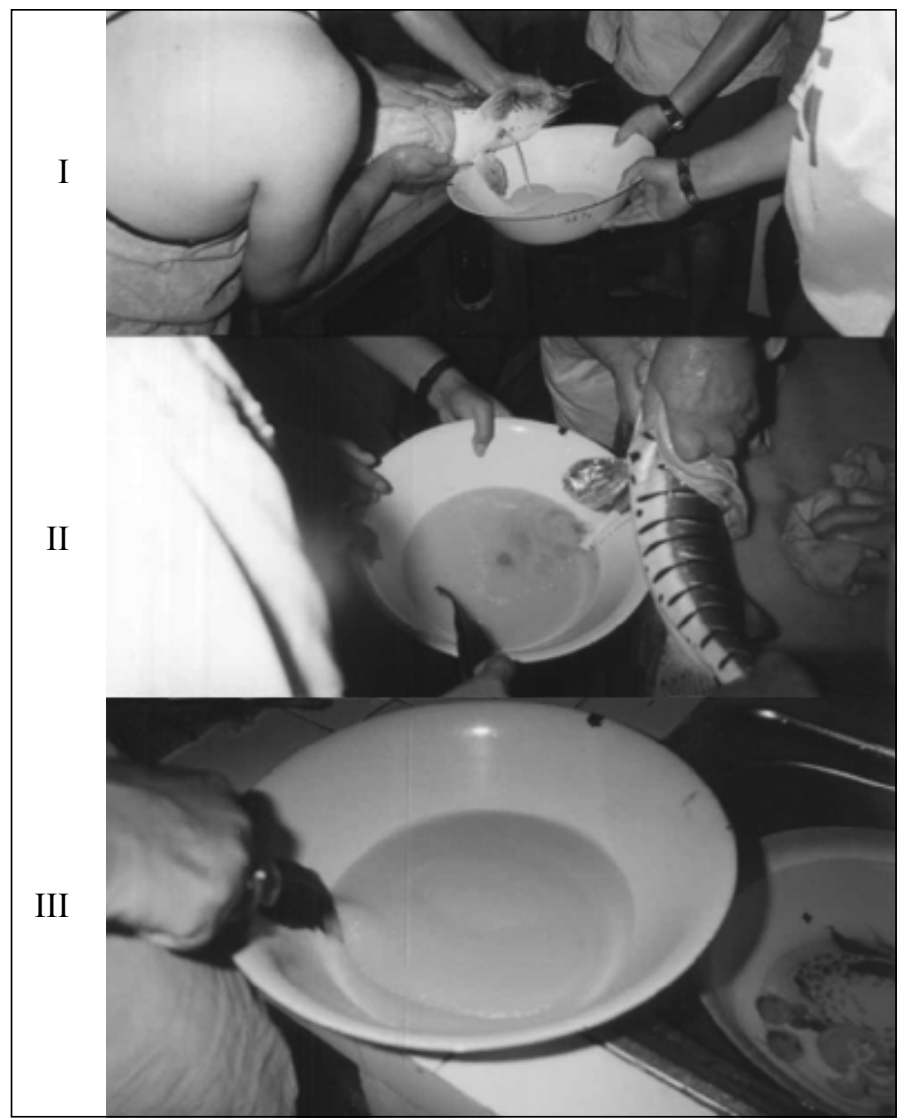


Tabla 1. Número de óvulos y larvas doncella, Pseudoplatystoma fasciatum, obtenidos a través de inducción hormonal.

\begin{tabular}{rrrrrrrr}
\hline Pez & $\begin{array}{r}\text { Peso del } \\
\text { pez (g) }\end{array}$ & $\begin{array}{r}\mathrm{N}^{\circ} \text { óvulos } \\
\text { por gramo }\end{array}$ & $\begin{array}{r}\text { Peso total } \\
\text { óvulos (g) }\end{array}$ & $\begin{array}{c}\mathrm{N}^{\circ} \text { Total } \\
\text { óvulos }\end{array}$ & $\mathrm{N}^{\circ}$ larvas & $\begin{array}{r}\% \text { de } \\
\text { larvas }\end{array}$ & Inductor \\
\hline 1 & 2165 & 1205 & 325 & 391625 & - & - & Pituitaria \\
2 & 2600 & 1200 & 285 & 342000 & - & - & Ovudal \\
3 & 3570 & 1200 & 370 & 444000 & 236716 & 53,3 & Ovudal \\
4 & 1990 & 1203 & 163 & 196089 & 20040 & 10,22 & Pituitaria \\
5 & 2395 & 1212 & 188 & 227856 & 193435 & 84,89 & Ovudal \\
$\Sigma$ & 12720 & 6020 & 1335 & 1606380 & 450191 & & - \\
$\mathrm{x}$ & 2544 & 1204 & 267 & 321276 & - & & - \\
\hline
\end{tabular}

Gráfico 1. Número de huevos en relación al peso de las hembras.

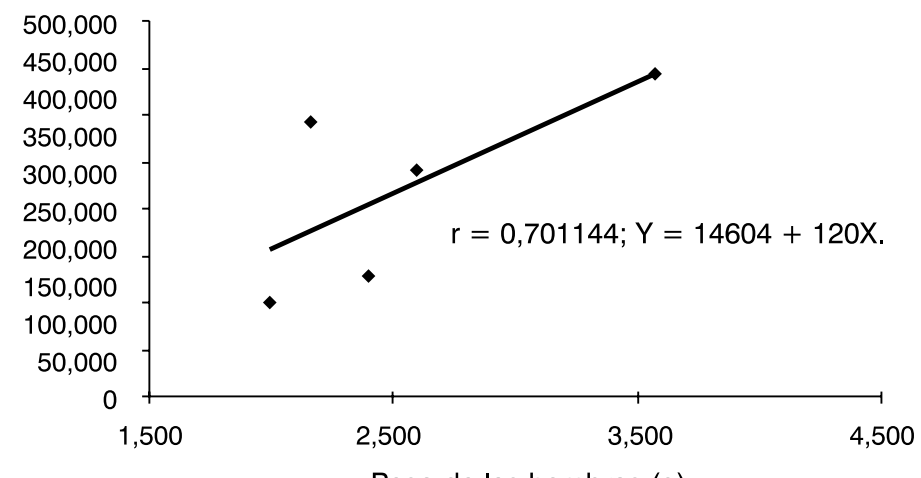

Peso de las hembras (g)

El diámetro de los óvulos hidratados, transparentes y semiflotantes, es de $8,5 \mathrm{~m}$. A los 15 minutos de incubación, se observa el espacio perivitelínico. La polarización es evidente a partir de los 27 minutos, con el polo animal o blastodisco en forma de bóveda semiesférica, montada sobre el periblasto que rodea a su vez el vitelo. Estas características corresponden a huevos telolecitos (acúmulo de vitelo en polo vegetal). 
En los siguientes 30 minutos, aparecen las divisiones de 8, 16 y 32 blastómeros. Luego, a los 135 minutos, se define el estado de mórula. A las 3 horas, se lleva a cabo la fase de clivaje, en la que culmina el estado de blástula.

Durante la cuarta hora, se observa la gastrulación, a partir de la cual se conforma el embrión temprano. Esta concluye con el cierre del blastóforo a las siete horas. En este periodo, la región cefálica se muestra diferenciada de la caudal y ya es apreciada la placa neural, que evidencia el estadio de néurula.

La formación de los miómeros comienza durante la octava hora; a partir de la misma, se hacen aparentes las vesículas ópticas. La cápsula ótica se observa a la undécima hora. Puede notarse el comienzo de la elongación de la región caudal cuando el embrión exhibe 12 miómeros. A la duodécima hora, se advierten los primeros movimientos autónomos y la formación de la aleta embrionaria, cuando se encuentran 20 miómeros. Durante la decimotercera hora, el embrión se mueve y gira constantemente sobre su propio eje (Figura 2).

Figura 2. Estadios de desarrollo del embrión.

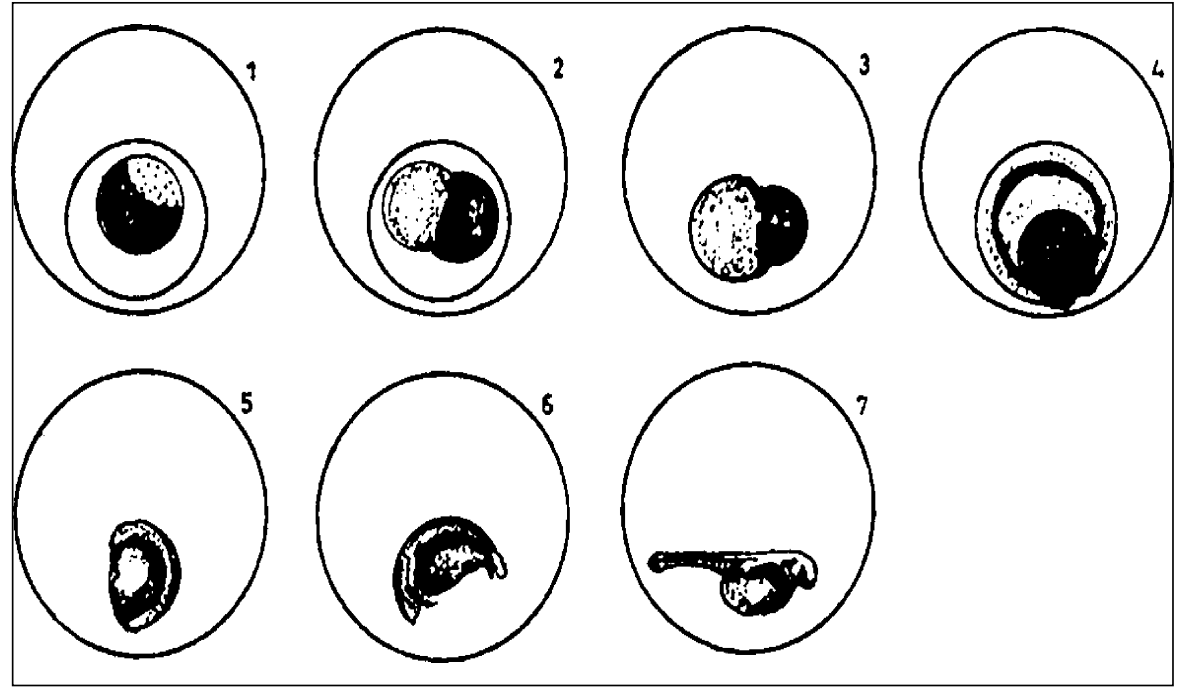

1. Huevo fecundado;

5. Fase de néurula;

2. Fase de mórula;

6. Embrión de 12 horas;

3. Fase de blástula;

7. Larva recién nacida.

4. Cierre del blastóporo; 
Durante la decimocuarta hora se inicia la eclosión. Al nacer, las larvas tienen una longitud total de 3,01 $\pm 0,12 \mathrm{~mm}$. En ese momento, las larvas exhiben el saco vitelino pigmentado y el extremo de la aleta embrionaria ligeramente heterocerca. Se cuenta entre 45 a 47 miómeros y se observa el punto oscuro de los ojos y la presencia de los otolitos.

Durante la hora vigésimo primera de vida, las larvas presentan los ojos pigmentados con los cristalinos formados. El saco vitelino se ha reducido a un $50 \%$ de su volumen y comienzan a desarrollarse los arcos branquiales y el primer par de barbellas (las maxilares).

A los dos días de edad, las larvas empiezan a tener movimientos mandibulares (boca abierta) y se observa el segundo par de barbellas (post-mentales). La vejiga natatoria se encuentra ya formada. El saco vitelino se reduce al $10 \%$ y se delinean los radios de la porción caudal en la aleta embrionaria.

A los tres días, la respiración branquial es más notoria a través de los movimientos rítmicos de los opérculos, los cuales cubren completamente cuatro pares de arcos branquiales donde se detallan las branquiespinas. Se verifica un cambio de conducta en las post-larvas. Estas tienden a concentrarse unas sobre otras en los sitios oscuros (preferentemente en las esquinas de los acuarios).

En el día cuarto, el saco vitelino se encuentra totalmente reabsorbido. El tubo digestivo se encuentra aparentemente completo y se observa los primeros movimientos peristálticos. En esta etapa, las post-larvas consumen su primer alimento vivo (plancton). El estómago se observa lleno. La longitud total de la larva es de $5,82 \pm 0,19 \mathrm{~mm}$.

A los cinco días, aparecen las proyecciones de las aletas pectorales y el tercer par de barbellas (sub-mentales).

A los seis días, se completa el desarrollo total de las barbellas, la diferenciación de la columna vertebral, la presencia de fibras musculares y la formación de la aleta caudal.

A los siete y ocho días de nacidas las post-larvas presentan todas sus estructuras anatómicas bien definidas.

Al noveno día, la pigmentación comienza a hacerse notar. Esta aparece, en primer lugar, en la parte de la cabeza. 
La actitud de las post-larvas es de reposo en el fondo. En la mayoría de los casos, se cambia súbitamente a rápidos movimientos rastreros cuando es suministrado el alimento vivo.

En el décimo día, se definen las características de coloración. En este periodo, se estima una longitud de 10,7mm (Figura 3).

Figura 3. Estadios de desarrollo de las larvas.

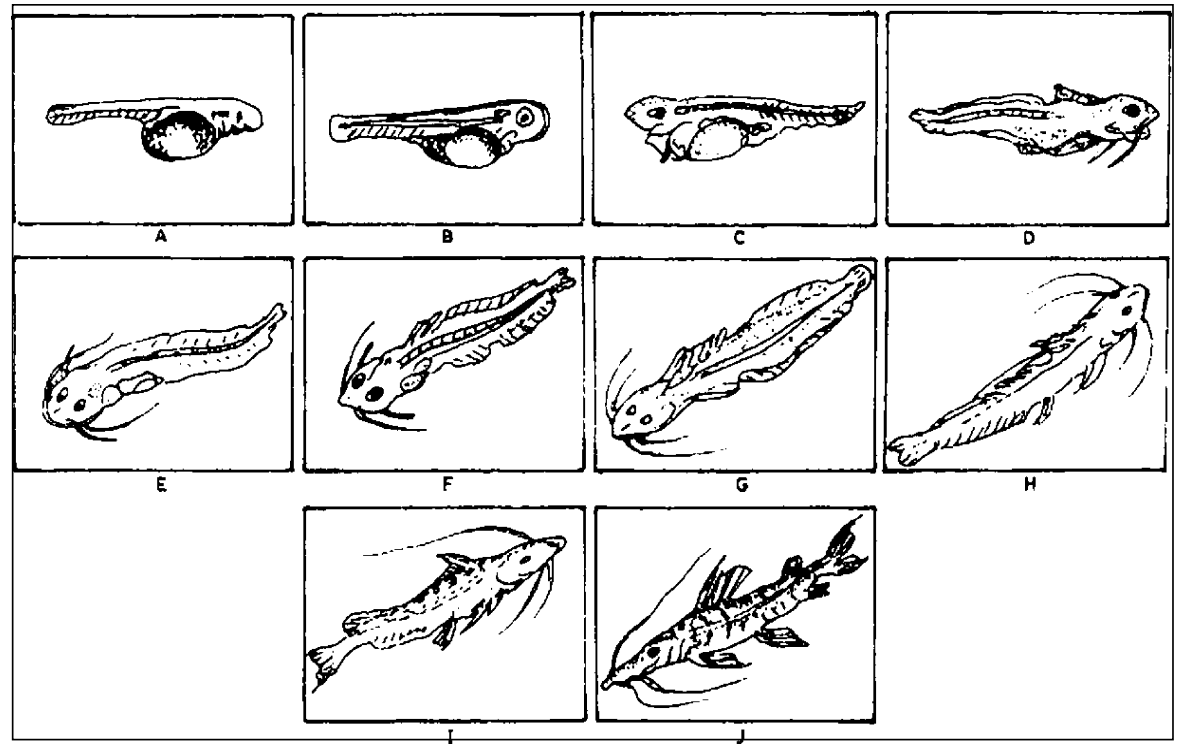
A. Larva de un día;
F. Larva de seis días;
B. Larva de dos días;
G. Larva de siete días;
C. Larva de tres días;
H. Larva de ocho días;
D. Larva de cuatro días;
I. Larva de nueve días;
E. Larva de cinco días;
J. Larva de diez días. 
Tabla 2. Parámetros físico-químicos del agua durante la fase de incubación de huevos de Pseudoplatystoma fasciatum.

\begin{tabular}{|c|c|c|c|c|}
\hline Hora & $\begin{array}{c}\text { Temperatura } \\
\left({ }^{\circ} \mathrm{C}\right)\end{array}$ & $\begin{array}{c}\text { Oxígeno disuelto } \\
(\mathrm{mg} / \mathrm{l})\end{array}$ & $\mathrm{pH}$ & Observaciones \\
\hline $19: 00$ & 27,0 & 3,9 & 5,8 & Inicio de la incubación \\
\hline $20: 00$ & 27,5 & 3,7 & 5,8 & \\
\hline $21: 00$ & 27,5 & 3,6 & 5,7 & \\
\hline $22: 00$ & 27,0 & 3,6 & 5,7 & \\
\hline $23: 00$ & 27,0 & 3,5 & 5,7 & \\
\hline $24: 00$ & 27,0 & 3,0 & 5,8 & \\
\hline $01: 00$ & 27,1 & 3,1 & 5,9 & \\
\hline $02: 00$ & 27,1 & 3,0 & 6,0 & \\
\hline $03: 00$ & 27,2 & 3,0 & 6,0 & \\
\hline $04: 00$ & 27,0 & 3,0 & 6,0 & \\
\hline $05: 00$ & 27,1 & 3,0 & 6,1 & \\
\hline $06: 00$ & 27,1 & 3,0 & 6,0 & \\
\hline $07: 00$ & 27,0 & 3,0 & 6,1 & \\
\hline $08: 00$ & 27,0 & 3,0 & 6,1 & \\
\hline $09: 00$ & 27,0 & 3,0 & 6,1 & Inicio de la eclosión \\
\hline $\mathrm{x}$ & 27,11 & 3,23 & 5,92 & \\
\hline $\mathrm{s}$ & 0,17 & 0,32 & 0,15 & \\
\hline
\end{tabular}

\section{DISCUSIÓN}

Son pocos los trabajos encontrados sobre la ontogenia y morfogénesis temprana de los silúridos sudamericanos. Ihering y Azevedo (1936) observaron que el desarrollo embrionario en dos especies de la familia Pimelodidae, Pimelodella lateristriga $y$ Rhamdia quelen, ocurría en un lapso de cuarenta y seis horas a una temperatura de 18 a $19^{\circ} \mathrm{C}$. Por otro lado, Godinho et al., (1978) reporta que en Rhamdia hilarii, otro pimelidido, el tiempo de eclosión es de 27 horas a una temperatura de $23 \pm 1^{\circ} \mathrm{C}$. 
Asimismo, Kossowski y Madrid (1986) manifiestan que Pseudoplatystoma fasciatum eclosiona a las 17 horas con 40 minutos de la fecundación, a una temperatura de $25^{\circ} \mathrm{C}$. Nosotros, en este trabajo, encontramos que Pseudoplatystoma fasciatum eclosiona a las $14 \pm 2$ horas de la fecundación a una temperatura de $27^{\circ} \mathrm{C}$. La corta duración de este proceso tiene semejanzas con algunas especies de lejana filogenia, pero de similares adaptaciones reproductivas de la familia Characidae, como Colossoma macropomum (Bermúdez et al. 1979) y Mylossoma duriventris (Kossowski, 1980).

Por otro lado, Lake (1967) manifiesta que los huevos con gran espacio perivitalínico hacen que el embrión esté más protegido contra los daños de las aguas torrenciales o flujos altos. En tal sentido, para P. fasciatum, que presenta huevos con gran espacio perivitelínico, se afirma que el desove puede ocurrir en los espacios turbios y abiertos de los grandes ríos durante las crecientes. Similar es lo que anuncia Goulding (1980) para C. macropomum y otros characidos.

Según Kossowski y Madrid (1986), el peculiar patrón de coloración inicial de las post-larvas es conservado durante dos meses y medio hasta tomar su coloración definida. Este es utilizado como una defensa que les permite camuflarse cuando son arrastradas a las riberas inundadas de los ríos. Igualmente, señala Mago (1970), en estos tipos de ambientes la vegetación semisumergida, como troncos y raíces de árboles, les proporciona sitios ideales para su ocultamiento. En estos lugares, se desarrolla una elevada productividad de zooplancton (daphnias, ciclops, etc.), de quironómidos, de larvas de insectos y de otros organismos que son susceptibles de ser consumidos por las post-larvas y larvas juveniles de Pseudoplatystoma fasciatum.

Asimismo, Reid (1983) manifiesta que Pseudoplatystoma fasciatum menores de 10 $\mathrm{cm}$, capturados en los ambientes antes descritos, estaban llenos de insectos acuáticos y de larvas de peces. A partir de ello, asevera que la característica de la aparición del patrón de coloración definitiva marcaría el inicio de los hábitos piscívoros de esta especie.

Por otra parte, Kossowski y Madrid (1986), manteniendo las larvas de Pseudoplatystoma fasciatun en acuarios de vidrio con ventilación constante y alimentadas con nauplius de Artemia salina, observaron un buen desarrollo larval. Estas larvas fueron sembradas a los 25 días de nacidas en tanques de concreto previamente tratados. Similarmente, nosotros en este trabajo colocamos 100 larvas en un acuario. Allí fueron alimentadas con zooplancton de los estanques y cápsulas de huevo a fin de observar su desarrollo, pero conseguimos mantenerlas solo por 10 días. 


\section{CONCLUSIONES}

- Se ha conseguido la reproducción artificial de la doncella Pseudoplatystoma fasciatum mediante la inducción hormonal usando pituitaria de carpa y ovudal (D-alanina 6).

- Se determinó que doncellas hembras tienen una media de 1204 óvulos por gramo de óvulos.

- Se encontró que esta especie crece muy bien en su primera fase larval (10 días) alimentada con zooplancton (daphnias, ciclops, etc.).

\section{BIBLIOGRAFÍA}

ALCÁNTARA, B.F.; GUERRA, F. H. 1986. Avances en la reproducción de alevinos de gamitana, Colossoma macropomum y paco C. brachypomum por reproducción inducida. En: Rev. Lat. Acui. ํ30-23-60. Lima (Perú).

BERMÚDEZ, D.; PRADA, N.; KOSSOWSKI, C. 1979. Ensayo sobre la reproducción de la cachama Colossoma macropomum (Cuvier) 1818, en cautiverio. En: Bol. Univ. Centro Occidental Barquisimeto (Venezuela). 23 pp.

CASTAGNOLLI, N. 1992. Criação de peixes de água doce. Campus de Jaboticabal, Faculdade de Ciências Agrárias e Veterinárias. FUNEP, 189 pp.

CANCINO, L. 1990. Efecto del extracto de pituitaria de carpa y de la hormona liberadora de gonadotropinas (LH-Rha) sobre la maduración gonadal del bagre rayado, Pseudoplatystoma fasciatum (Linnaeus 1766) (Pisces. Siluriformes). Universidad Jorge Tadeo Lozano, Facultad de Biología Marina. Tesis de grado. $87 \mathrm{pp}$.

CONTRERAS, P.; CONTRERAS, J. 1989. Resultados preliminares de la reproducción inducida del bagre rayado, Pseudoplatystoma fasciatum (Linnaeus, 1766). Proyecto estación piscícola San Silvestre. En: Inderena Barrancabermeja. pp. 13-21.

GODINHO, H.; FENERICH, N.; NARAHARA, M. 1978. Desenvolvimento embrionario e larval de Rhamdia hilarii (Valencienses, 1840). (Siluriformes, Pimelodidae). En: Rev. Brazil. Biol. 38 (1): 151-156. 
GOULDING, M. 1980. The Fishes and the Forest, Explorations in Amazonian Natural History. Univ. of California Press, Berkeley, $280 \mathrm{pp}$.

IHERING, R.; AZEVEDO, P. 1936. A desova e a hipofisação dos peixes. Evolução de dois nematognathas. En: Arq. Inst. Biol. S. Paulo, 7: 107-118.

KOSSOWSKI, C. 1980. Ensayo de la reproducción inducida en palometa carachita Mylossoma duriventris (Cuvier) 1818 (Pisces, Cipriniformes) con uso de gonadotropina corionica humana. En: Acta Científica Venezolana 31: 444448 .

KOSSOWSKI, C.; MADRID, F. 1985. Ensayo de la reproducción inducida en el bagre rayado cabezón Pseudoplatystoma fasciatum (Linnaeus 1766) (Pisces. Siluriformes). En: Acta Científica Venezolana 36: 284-285.

KOSSOWSKI, C.; MADRID, F. 1986. Observaciones de los estadios embrionario y larval del «bagre rayado cabezón» Pseudoplatystoma fasciatum (Linnaeus, 1766) (Pisces. Siluriformes). Estación de piscicultura, Escuela de Agronomía. UCLA, Barquisimeto (Venezuela).

LAKE. J. 1967. Rearing experiments with species of Australian, freshwater fishes. Aust. J. Mar. Freshwater Res. 18:155-173 pp.

LOPES, M.C.; FREIRE, R.A.B.; VICENSOTTO, J.R.M.; SENHORINI, J.A. 1996. Alimentação de larvas de surubim pintado, Pseudoplatystoma coruscans (Agassiz,1829), em laboratório, na primeira semana. En: Bol. Tec. CEPTA. Pirassununga, vol, 9 p 11-21.

MAGO, F. 1970. Estudios preliminares sobre la ecología de los peces de los Llanos de Venezuela. En: Acta Biol. Venez. 7(1):71-102.

REID, S. 1983. La biología de los bagres rayados Pseudoplatystoma fasciatum Pseudoplatystoma tigrinum en la cuenca del río Apuré, Venezuela. En: Revista UNELLEZ de Ciencia y Tecnología Producción Agrícola. (1):13-41.

ROTHBARD, S. 1981. Induced Reproduction in Cultivated Cyprinids. The Common Carp and the Group of Chinese Carp. I. The Technique of Induction, Spawing and Hatching. En: Bamidgeh Vol. 33 N.4, 103-121. 
WOYNAROVICH, E.; HORVATH, H. 1981. Propagación artificial de peces de aguas templadas: Manual para extensionistas. FAO. En:Doc.Tec. Pesca 187-201pp.

WOYNAROVICH, A.; WOYNAROVICH, E. 1998. Reproducción artificial de las especies Colossoma y Piaractus. Una guía detallada para la reproducción de alevinos de gamitana, paco y caraña. Fondo Nacional de Desarrollo Pesquero-Fondepes. $1^{\circ}$ Edición. Lima (Perú). 67 pp. 